\title{
Numerical-perturbation technique for the transverse vibrations of highly prestressed plates
}

\author{
Ali H. Nayfeh and M. P. Kamat \\ Department of Engineering Science and Mechanics, Virginia Polytechnic Institute and State University, \\ Blacksburg, Virginia 24061 \\ (Received 11 June 1975; revised 2 June 1976)

\begin{abstract}
Under the usual assumptions of small strains with moderately large rotations, the problem of the transverse vibrations of highly prestressed, nonuniform annular plates is reduced to the solution of the differential equation governing the transverse vibration of the corresponding prestressed membrane subject to modified boundary conditions that account for the effects of bending. The methods of matched asymptotic and/or composite expansions are used to determine these modified boundary conditions. The agreement of the results of both methods with known exact solutions for simple geometries demonstrates the efficiency of this technique when compared with other well-known numerical techniques.
\end{abstract}

PACS numbers: 43.40.Dx

\section{INTRODUCTION}

The exact solution to the problem of the transverse vibrations of a uniformly thick, uniformly prestressed annular plate is well known. ${ }^{1}$ The solution is in terms of regular and modified Bessel functions of the first and second kind. However, in the case of nonuniform plates with in-plane forces, closed form solutions do not exist even for circular or annular geometries, and recourse has to be made to approximate techniques such as numerical methods, perturbation methods, or combinations of both.

When the applied loads are large compared with $D_{0}^{*} / a_{0}^{* 2}$, where $D_{0}^{*}$ is the rigidity and $a_{0}^{*}$ is a characteristic length of the plate, that is, $\epsilon=\left(D_{0}^{*} / N^{*} a_{0}^{* 2}\right)^{1 / 2} \ll 1$, where $N$ is a measure of the applied loads, a state of membrane deformation prevails over the plate except in thin layers next to the edges of the plate where a state of bending deformation exists. As $\in$ decreases, these bending layers become thinner.

With a purely numerical technique, such as the finiteelement method, ${ }^{2}$ the entire plate is modeled using a large number of bending elements to accurately simulate the effects of the boundary conditions on the displacement and stress distributions, thereby not exploiting the fact that a large portion of the plate behaves like a prestressed membrane. As $\in \rightarrow 0$, more and more elements will be needed, resulting in a large computational time.

On the other hand, purely analytical methods are capable of yielding solutions for simple geometries. To treat the vibration of highly prestressed plates with complex geometries, we propose a numerical-perturbation technique. This technique is demonstrated for annular nonuniform plates.

The perturbation methods of matched asymptotic and/ or composite expansions are used to reduce the solution of the problem of the transverse vibrations of nonuniform, highly prestressed plates to the solution of the simpler problem of the transverse vibrations of non- uniform membranes, but with modified boundary conditions that account for the effects of bending. This simpler problem is then solved by using a finite-element method. It should be mentioned that only the geometric matrix is needed in a membrane element (no bending rigidity), while the stiffness as well as the geometric matrices are needed in a bending element to model the effects of in-plane forces on out-of-plane deformations. Moreover, since the governing equation in the case of a vibrating membrane is a second-order, partial differential equation, the geometric matrix for this analysis can be based upon a lower-order polynomial for the out-of-plane displacement function than that which would be used for vibrating plates. This then leads to a reduction in the size of the geometric matrix for each element, which in turn implies a reduction of the total number of degrees of freedom of the assembled model.

\section{PROBLEM FORMULATION}

We consider linear transverse vibrations of nonuniform, highly prestressed, annular plates. For infinitesimal transverse vibrations, the effects of midplane stretching on the in-plane loads can be neglected.

We introduce dimensionless quantities by using the outer radius $a_{0}^{*}$, a characteristic thickness $h_{0}^{*}$ at a specified position, a characteristic period $T^{*}$, and a characteristic load $N^{*}$ as reference quantities. Thus, if starred and unstarred quantities denote dimensional and dimensionless quantities, we have

$$
\begin{array}{lll}
w=w^{*} / a_{0}^{*}, & a=a_{i}^{*} / a_{0}^{*}, & t=t * / T^{*}, \\
r=r^{*} / a_{0}^{*}, & h=h^{*} / h_{0}^{*}, & N_{r r}=N_{r r}^{*} / N^{*}, \\
N_{\theta \theta} \doteq N_{\theta \theta}^{*} / N^{*}, & N_{r \theta}=N_{r \theta}^{*} / N^{*}, &
\end{array}
$$

where $w$ is the transverse displacement, $a_{i}^{*}$ is the inner radius, $t$ is the time, $r$ is the radial distance, $h$ is the thickness, and the $N_{i j}$ are the in-plane load distributions. Under the above assumptions and in terms of these dimensionless variables, the equation describing the transverse vibrations of a plate having a nonuniform thickness is ${ }^{4}$ 


$$
\begin{aligned}
& \epsilon^{2} \nabla^{2}\left(h^{3} \nabla^{2} w\right)-\left[N_{r r} \frac{\partial^{2} w}{\partial r^{2}}+N_{\theta \theta}\left(\frac{1}{r} \frac{\partial w}{\partial r}+\frac{1}{r^{2}} \frac{\partial^{2} w}{\partial \theta^{2}}\right)+2 N_{r \theta}\left(\frac{1}{r} \frac{\partial^{2} w}{\partial r \partial \theta}-\frac{1}{r^{2}} \frac{\partial w}{\partial \theta}\right)\right]+\alpha^{2} h \frac{\partial^{2} w}{\partial t^{2}} \\
& -\epsilon^{2}(1-\nu)\left\{\frac{\partial^{2}}{\partial r^{2}}\left(h^{3}\right)\left(\frac{1}{r} \frac{\partial w}{\partial r}+\frac{1}{r^{2}} \frac{\partial^{2} w}{\partial \theta^{2}}\right)+\frac{\partial^{2} w}{\partial r^{2}}\left[\frac{3 h^{2}}{r} \frac{\partial h}{\partial r}+\frac{1}{r^{2}} \frac{\partial^{2}}{\partial \theta^{2}}\left(h^{3}\right)\right]+2\left[\frac{1}{r} \frac{\partial^{2}}{\partial r \partial \theta}\left(h^{3}\right)-\frac{3 h^{2}}{r^{2}} \frac{\partial h}{\partial \theta}\right]\left(\frac{1}{r} \frac{\partial^{2} w}{\partial r \partial \theta}-\frac{1}{r^{2}} \frac{\partial w}{\partial \theta}\right)\right\}=0
\end{aligned}
$$

where

$$
\epsilon^{2}=D_{0}^{*} / N * a_{0}^{*}, \quad \alpha^{2}=\rho^{*} a_{0}^{* 2} h_{0}^{*} / N * T *^{2},
$$

with $\rho^{*}$ being the mass per unit volume. The in-plane loads $N_{r r}, N_{r \theta}$, and $N_{\theta \theta}$ are assumed to be known functions of $r$ and $\theta$; they are solutions of the plane-stress problem.

To complete the problem formulation, we need to specify the boundary conditions. For definiteness, we consider the case of a clamped annular plate for which

$$
w=0 \text { and } \frac{\partial w}{\partial r}=0 \text { at } r=a \text { and } r=1 \text {, }
$$

where $a<1$. For a circular plate, the boundary condition at $r=a$ is replaced by

$$
w<\infty \text { at } r=0 \text {. }
$$

\section{REDUCTION tO A MEMBRANE PROBLEM}

For highly prestressed plates, $N^{*}$ is large compared with $D_{0}^{*} a_{0}^{*}$, so that $\epsilon \ll 1$. Consequently, one is tempted to determine a straightforward perturbation expansion for $w$ in powers of $\epsilon$ in the form

$$
w(r, \theta, t ; \epsilon)=w_{0}(r, \theta, t)+\epsilon w_{1}(r, \theta, t)+\epsilon^{2} w_{2}(r, \theta, t)+\cdots .
$$

Substituting this expansion in Eq. (2) and equating coefficients of like powers of $\epsilon$ leads to

$$
\begin{aligned}
\mathscr{L}\left(w_{0}\right) \equiv & N_{r r} \frac{\partial^{2} w_{0}}{\partial r^{2}}+N_{\theta \theta}\left(\frac{1}{r} \frac{\partial w_{0}}{\partial r}+\frac{1}{r^{2}} \frac{\partial^{2} w_{0}}{\partial \theta^{2}}\right) \\
& +2 N_{r \theta}\left(\frac{1}{r} \frac{\partial^{2} w_{0}}{\partial r \partial \theta}-\frac{1}{r^{2}} \frac{\partial w_{0}}{\partial \theta}\right)-\alpha^{2} h \frac{\partial^{2} w_{0}}{\partial t^{2}}=0, \\
\mathcal{L}\left(w_{1}\right)= & 0, \\
\mathcal{L}\left(w_{2}\right) & =F_{2}\left(w_{0}\right), \\
\mathcal{L}\left(w_{n}\right) & =F_{n}\left(w_{n-2}\right) \text { for } n>2,
\end{aligned}
$$

where the $F_{n}$ are known functions of the $w_{n-2}$.

We note that the equations governing the $w_{n}$ are of second order as opposed to the original Eq. (2) which is of fourth order. Hence, the expansion (6) cannot be expected to satisfy, in general, all the boundary conditions, and consequently, it is not valid near the plate edges. Physically, for small $\epsilon$, a state of membrane deformation exists everywhere in the plate except in thin layers near the edges where bending deformations exist. These layers are usually called edge layers. Across these layers, the displacement changes very rapidly from a state of membrane deformation to a state of bending deformation in order to satisfy the boundary conditions. ${ }^{5,6}$ To determine an expansion that is uniformly valid, one can use ${ }^{3}$ the method of multiple scales, the method of matched asymptotic expansions, or the method of composite expansions. In this paper, we discuss the latter two methods.

According to the method of matched asymptotic expansions, one determines expansions, which we refer to as bending expansions, that are valid in the edge layers by using stretched transformations. Then, these expansions are matched to the straightforward expansion (6) (membrane expansion). A uniformly valid expansion is subsequently formed by adding the bending expansions to the membrane expansion and subtracting the common parts. In this problem, the stretching transformation near the outer edge is

$$
\xi_{0}=(1-r) / \epsilon,
$$

while the stretching transformation near the inner edge is.

$$
\xi_{i}=(r-a) / \epsilon .
$$

For a discussion on determination of appropriate stretching transformations, see, for example, Sec. 4.1.2 of Ref. 3. Consequently, the first-order composite expansion that is uniformly valid has the form

$$
\begin{aligned}
w(r, \theta, t ; \epsilon)= & \sum_{j=0}^{1} \epsilon^{i} w^{(m)}(r, \theta, t, \epsilon)+\sum_{j=0}^{1} \epsilon^{j} w_{j}^{(0)}\left(\xi_{0}, \theta, t\right) \\
& +\sum_{j=0}^{1} \epsilon^{j} w_{j}^{(i)}\left(\xi_{i}, \theta_{i}, t\right)+O\left(\epsilon^{2}\right),
\end{aligned}
$$

where $w^{(m)}$ satisfies the membrane, Eq. (7), and

$$
w_{j}^{(0)}\left(\xi_{0}, \theta, t\right) \rightarrow 0 \text { as } \xi_{0} \rightarrow \infty
$$

and

$$
w_{j}^{(i)}\left(\xi_{i}, \theta, t\right) \rightarrow 0 \text { as } \xi_{i} \rightarrow \infty, \quad j=0 \text { and } 1 .
$$

In other words, $w_{j}^{(0)}$ is significant only in the thin bending layer next to the outer edge, while $w_{j}^{(i)}$ is significant in the thin bending layer next to the inner edge.

\section{A. Method of matched asymptotic expansion}

According to this method, we seek an outer expansion for $w$ of the form

$$
w^{(m)}=w_{0}^{(m)}(r, \theta, t)+\epsilon w_{1}^{(m)}(r, \theta, t)+\cdots .
$$

Substituting Eq. (15) into Eq. (2) and equating coefficients of like powers of $\epsilon$, we find that $w_{0}^{(0)}+\epsilon w_{1}^{(0)}$ satisfy

$$
\begin{aligned}
N_{r r} \frac{\partial^{2} w}{\partial r^{2}} & +N_{\theta \theta}\left(\frac{1}{r} \frac{\partial w}{\partial r}+\frac{1}{r^{2}} \frac{\partial^{2} w}{\partial \theta^{2}}\right)+2 N_{r \theta}\left(\frac{1}{r} \frac{\partial w}{\partial r \partial \theta}-\frac{1}{r^{2}} \frac{\partial w}{\partial \theta}\right) \\
& -\alpha^{2} h \frac{\partial^{2} w}{\partial t^{2}}+O\left(\epsilon^{2}\right)=0 .
\end{aligned}
$$

\section{Inner expansion near $r=1$}

The boundary layer at $r=1$ is characterized by the stretching transformation, Eq. (11). In this case, we seek an expansion of the form 


$$
w^{(i)}=w_{0}^{(i)}\left(\xi_{0}, \theta, t\right)+\epsilon w_{1}^{(i)}\left(\xi_{0}, \theta, t\right)+\cdots .
$$

Writing the original equation, Eq. (2), in terms of the stretched variable $\xi_{0}$, substituting for $w^{(i)}$ from Eq. (17), expanding for small $\epsilon$, and collecting coefficients of powers of $\epsilon$, we have

$$
\frac{\partial^{4} w_{0}^{(i)}}{\partial \xi_{0}^{4}}-\lambda_{r r}^{2}(1, \theta) \frac{\partial^{2} w_{0}^{(i)}}{\partial \xi_{0}^{2}}=0
$$

and

$$
\begin{aligned}
\frac{\partial^{4} w_{1}^{(i)}}{\partial \xi_{0}^{4}}- & \lambda_{r r}^{2}(1, \theta) \frac{\partial^{2} w_{1}^{(i)}}{\partial \xi_{0}^{2}}=2 \frac{\partial^{3} w_{0}^{(i)}}{\partial \xi_{0}^{3}}-\lambda_{\theta \theta}^{2}(1, \theta) \frac{\partial w_{0}^{(i)}}{\partial \xi_{0}} \\
& -2 \lambda_{r \theta}^{2}(1, \theta) \frac{\partial^{2} w_{0}^{(i)}}{\partial \xi_{0} \partial \theta}
\end{aligned}
$$

where

$$
\lambda_{i j}^{2}(1, \theta)=N_{i j}(1, \theta) h^{-3}(1, \theta) .
$$

The general solution of Eq. (18a) is

$w_{0}^{(i)}=\alpha_{1}(\theta, t)+\alpha_{2}(\theta, t) \xi_{0}+\alpha_{3}(\theta, t) e^{-\lambda r r \xi_{0}}+\alpha_{4}(\theta, t) e^{\lambda_{r} r_{0}}$.

The constant $\alpha_{4}$ must be zero; otherwise $w_{0}^{(i)}$ would grow exponentially with $\xi_{0}$, and it could not be matched with the outer expansion. The general solution of Eq. $(18 \mathrm{~b})$ is

$$
\begin{aligned}
w_{1}^{(i)}= & \alpha_{5}(\theta, t)+\alpha_{6}(\theta, t) \xi_{0}+\alpha_{7}(\theta, t) e^{-\lambda_{r r} \xi_{0}}+\alpha_{8}(\theta, t) e^{\lambda_{r r} \xi_{0}} \\
& -\alpha_{9}(\theta, t) \xi_{0}^{2} e^{-\lambda_{r r} \xi_{0}}-\alpha_{10}(\theta, t) \lambda_{r r}^{-3}\left(1+\xi_{0}\right) e^{-\lambda_{r r} \xi_{0}} \\
& -\frac{1}{2} \alpha_{2}(\theta, t)\left(\lambda_{r r} / \lambda_{\theta \theta}\right)^{2} \xi_{0}^{2},
\end{aligned}
$$

where

$$
\begin{aligned}
\alpha_{9}= & \frac{1}{4} \alpha_{3} \lambda_{r r} \frac{\partial \lambda_{r r}}{\partial \theta}, \\
\alpha_{10}= & \frac{1}{2}\left(-2 \alpha_{3} \lambda_{r r}^{3}+\alpha_{3} \lambda_{\theta \theta}^{2} \lambda_{r r}-\frac{\partial \alpha_{3}}{\partial \theta} \lambda_{r r}-\alpha_{3} \frac{\partial \lambda_{r r}}{\partial \theta}\right) \\
& +\frac{5}{4}\left(\alpha_{3} \lambda_{r r} \frac{\partial \lambda_{r r}}{\partial \theta}\right) .
\end{aligned}
$$

Again $\alpha_{8}$ must be zero; otherwise $w_{1}^{(i)}$ would grow exponentially with $\xi_{0}$, and it could not be matched with the outer expansion. The functions $\alpha_{1}, \alpha_{2}, \ldots, \alpha_{7}$ have to be related from the boundary conditions

$$
\left.\begin{array}{l}
w_{0}^{(i)}=0 ; w_{1}^{(i)}=0 \\
\frac{\partial w_{0}^{(i)}}{\partial \xi_{0}}=0 ; \frac{\partial w_{1}^{(i)}}{\partial \xi_{0}}=0
\end{array}\right\} \text { at } r=1 \text { or } r=a .
$$

Substituting these boundary conditions into Eqs. (19) and $(20)$, we have

$$
\begin{aligned}
& \alpha_{1}+\alpha_{3}=0, \\
& \alpha_{2}-\lambda_{r r} \alpha_{3}=0, \\
& \alpha_{5}+\alpha_{7}=0, \\
& \alpha_{5}-\lambda_{r r} \alpha_{6}=0 .
\end{aligned}
$$

Thus,

$$
\begin{aligned}
w_{0}^{(i)}= & \alpha_{1}\left(1-\lambda_{r r} \xi_{0}-e^{-\lambda r r^{\xi}}\right), \\
w^{(i)}= & \alpha_{5}\left(1-\lambda_{r r} \xi_{0}-e^{-\lambda_{r r} \xi_{0}}\right)-\alpha_{9} \xi_{0}^{2} e^{-\lambda_{r r} \xi_{0}}-\frac{1}{2} \alpha_{2}\left(\lambda_{r r} / \lambda_{\theta \theta}\right)^{2} \xi_{0}^{2} \\
& -\alpha_{10} \lambda_{r r}^{-3}\left(1+\xi_{0}\right) e^{-\lambda_{r r} \xi_{0}} .
\end{aligned}
$$

\section{Matching}

Two-term inner (two-term outer) equals two-term outer (two-term inner). Two-term outer equals

$$
w_{0}^{(m)}(r, \theta, t)+\epsilon w_{1}^{(m)}(r, \theta, t)+\cdots
$$

Rewritten in inner variable it equals

$$
w_{0}^{(m)}\left(1-\epsilon \xi_{0}, \theta, t\right)+\epsilon w_{1}^{(m)}\left(1-\epsilon \xi_{0}, \theta, t\right)+\cdots .
$$

expanded for small $\epsilon$ it equals

$$
w_{0}^{(m)}(1, \theta, t)+\epsilon\left[w_{1}^{(m)}(1, \theta, t)-\xi_{0} w_{0}^{(m) \prime}(1, \theta, t)\right]+\cdots,
$$

Two-term inner of two-term outer equals

$$
w_{0}^{(m)}(1, \theta, t)+\epsilon\left[w_{1}^{(m)}(1, \theta, t)-\xi_{0} w_{0}^{(m) \prime}(1, \theta, t)\right]_{+} \cdots .
$$

Two-term inner equals

$$
\begin{gathered}
\alpha_{1}\left(1-\lambda_{r r} \xi_{0}-e^{-\lambda_{r r} \xi_{0}}\right)+\alpha_{5} \epsilon\left[1-\lambda_{r r} \xi_{0}-e^{\left(-\lambda_{r r} \xi_{0}\right)}\right] \\
-\alpha_{9} \xi_{0}^{2} \epsilon e^{-\lambda_{r r} \xi_{0}}-\alpha_{10} \lambda_{r r}^{-3}\left(1+\xi_{0}\right) \epsilon e^{-\lambda_{r r} \xi_{0}} .
\end{gathered}
$$

Rewritten in the outer variable it equals

$$
\begin{aligned}
& \alpha_{1}\left\{1-\lambda_{r r} \frac{(1-r)}{\epsilon}-e^{-\lambda_{r r}} \frac{(1-r)}{\epsilon}\right\} \\
& \quad+\epsilon \alpha_{5}\left\{1-\lambda_{r r} \frac{(1-r)}{\epsilon}-e^{-\lambda_{r r}} \frac{(1-r)}{\epsilon}\right\}-\frac{1}{2} \epsilon \alpha_{1} \lambda_{r r}\left(\frac{1-r}{\epsilon}\right)^{2} \\
& \quad-\epsilon \alpha_{9}\left(\frac{1-r}{\epsilon}\right)^{2} e^{-\lambda r r}\left(\frac{1-r}{\epsilon}\right)-\epsilon \alpha_{10} \lambda_{r r}^{-3}\left(1+\frac{1-r}{\epsilon}\right) \\
& \quad \times \exp \left[-\lambda_{r r}\left(\frac{1-r}{\epsilon}\right)\right],
\end{aligned}
$$

Two-term outer of two-term inner equals

$-\frac{\alpha_{1}}{\epsilon} \lambda_{r r}(1-r)-\frac{\alpha_{1}}{2 \epsilon} \lambda_{r r}(1-r)^{2}+\alpha_{1}-\alpha_{5} \lambda_{r r}(1-r)+\epsilon \alpha_{5}+$ e.s.t.

where e.s.t. denotes "exponentially small terms." Using the matching principle we have

$$
\begin{aligned}
& \alpha_{1} \equiv 0, \\
& w_{0}^{(m)}(1, \theta, t)=\alpha_{1}, \\
& w_{1}^{(m)}(1, \theta, t)=\alpha_{5}, \\
& w_{0}^{\prime(m)}(1, \theta, t)=\alpha_{5} \lambda_{r r} .
\end{aligned}
$$

Equations (23a)-(23c) imply that

$$
\begin{aligned}
& w_{0}^{(m)}(1, \theta, t)=0, \\
& w_{1}^{(m)}(1, \theta, t)=\left(1 / \lambda_{r r}\right) w_{0}^{\prime(m)}(1, \theta, t) .
\end{aligned}
$$

Equations (24) are the boundary conditions subject to which Eq. (15) has to be solved for the outer expansion $w^{(m)}$.

\section{Assuming that}

$$
w(r, \theta, t)=\phi(r, \theta) e^{(-i \omega t)},
$$

we rewrite Eq. (16) as

$$
L \phi+\lambda \phi=0,
$$

where

$$
\begin{aligned}
& L=N_{r r} \frac{\partial^{2}}{\partial r^{2}}+N_{\theta \theta}\left(\frac{1}{r} \frac{\partial}{\partial r}+\frac{1}{r} \frac{\partial}{\partial \theta^{2}}\right)+2 N_{r \theta}\left(\frac{1}{r} \frac{\partial^{2}}{\partial r \partial \theta}-\frac{1}{r^{2}} \frac{\partial}{\partial \theta}\right) \\
& \lambda=\alpha^{2} \omega^{2} h .
\end{aligned}
$$


Putting

$$
\phi(r, \theta)=\phi_{0}(r, \theta)+\epsilon \phi_{1}(r, \theta)+\cdots
$$

in Eq. (25) and equating coefficients of like powers of $\epsilon$ yields

$$
\begin{aligned}
& L \phi_{0}+\lambda \phi_{0}=0, \\
& L \phi_{1}+\lambda \phi_{1}=0 .
\end{aligned}
$$

Similarly, the boundary conditions (24) become

$$
\begin{aligned}
& \phi_{0}(1, \theta)=0, \\
& \phi_{1}(1, \theta)=\left(1 / \lambda_{r r}\right) \phi_{0}^{\prime}(1, \theta) .
\end{aligned}
$$

It is immediately obvious that the homogeneous Eq. (26) subject to boundary conditions (27) can have only the trivial solution $\phi_{1} \equiv 0$, unless we expand the eigenvalue $\lambda$ as a function of $\epsilon$; that is

$$
\lambda=\lambda_{0}+\epsilon \lambda_{1}+\cdots \text {. }
$$

Thus, we have

$$
\begin{aligned}
& L \phi_{0}+\lambda_{0} \phi_{0}=0, \\
& L \phi_{1}+\lambda_{0} \phi_{1}=-\lambda_{1} \phi_{0} .
\end{aligned}
$$

Equation (28a) is a homogeneous equation subject to homogeneous boundary condition (27a). This is an eigenvalue problem which yields an infinite set of eigenvalues $\lambda_{0 m n}$ and eigenfunctions $\phi_{0 m n}$. Equation (28b) can then be solved subject to boundary condition $(27 \mathrm{~b})$ by expressing $\phi_{1}$ as a linear combination of these eigenfunctions. This latter equation enables the calculation of $\lambda_{1}$ which, when multiplied by $\epsilon$, yields the required correction to $\lambda_{0}$.

\section{B. Method of composite expansions}

According to the method of composite expansions, one assumes an expansion of the form (13) where $\xi_{0}$ and $\xi_{i}$ are defined by Eqs. (11) and (12), and $w^{(0)}$ and $w^{(i)}$ satisfy the conditions (14). To determine $w^{(0)}$, we use the variable 11 instead of $r$ and express Eq. (13) as

$$
\begin{aligned}
w= & w^{(m)}\left(1-\epsilon \xi_{0}, \theta, t\right)+\sum_{j=0}^{1} \epsilon^{j} w_{j}^{(0)}\left(\xi_{0}, \theta, t\right) \\
& +\sum_{j=0}^{1} \epsilon^{j} w_{j}^{(i)}\left(\frac{1-\epsilon \xi_{0}-a}{\epsilon}, \theta, t\right)+O\left(\epsilon^{2}\right) .
\end{aligned}
$$

Expanding Eq. (29) for small $\epsilon$ with $\xi_{0}$ being fixed and recalling that $w^{(i)} \rightarrow 0$ as $\xi_{i} \rightarrow \infty$, we obtain

$$
\begin{aligned}
w= & w^{(m)}(1, \theta, t)-\epsilon \xi_{0} \frac{\partial w^{(m)}}{\partial r}(1, \theta, t)+w_{0}^{(0)}\left(\xi_{0}, \theta, t\right) \\
& +\epsilon w_{1}^{(0)}\left(\xi_{0}, \theta, t\right)+O\left(\epsilon^{2}\right) .
\end{aligned}
$$

Changing the independent variable $r$ in Eq. (2) to $\xi_{0}$, substituting for $w$ into the resulting equation, and equating coefficients of like powers of $\epsilon$, we have

$$
\begin{aligned}
& \frac{\partial^{4} w_{0}^{(0)}}{\partial \xi_{0}^{4}}-\lambda_{r r}^{2}(1, \theta) \frac{\partial^{2} w_{0}^{(0)}}{\partial \xi_{0}^{2}}=0, \\
& \frac{\partial^{4} w_{1}^{(0)}}{\partial \xi_{0}^{4}}-\lambda_{r r}^{2}(1, \theta) \frac{\partial^{2} w_{1}^{(0)}}{\partial \xi_{0}^{3}}=2 \frac{\partial^{3} w_{0}^{(0)}}{\partial \xi_{0}^{3}}-\lambda_{\theta \theta}^{2}(1, \theta) \frac{\partial w_{0}^{(0)}}{\partial \xi_{0}} \\
& +2 \lambda_{r \theta}^{2}(1, \theta) \frac{\partial^{2} w_{0}^{(0)}}{\partial \xi \partial \theta} .
\end{aligned}
$$

The solutions of Eqs. (31) and (32) that tend to zero as $\xi_{0} \rightarrow \infty$ are

$$
\begin{aligned}
w_{0}^{(0)} & =c_{0}(\theta, t) e^{-\lambda_{r} \xi_{0}} \\
w_{1}^{(0)} & =c_{1}(\theta, t) e^{-\lambda_{r r} \xi_{0}}+c_{2} \lambda_{\lambda \lambda}^{-3}\left(1+\xi_{0}\right) e^{-\lambda r \xi_{0}} \\
& +c_{3} \xi_{0}^{2} e^{-\lambda_{r} \xi_{0}},
\end{aligned}
$$

where $c_{0}, c_{1}, c_{2}$, and $c_{3}$ are still unknown functions. Similarly, one can show that

$w_{0}^{(i)}=b_{0}(\theta, t) e^{-\beta_{r} \xi_{0}}$,

$w_{1}^{(i)}=b_{1}(\theta, t) e^{-\beta_{r r} \xi_{0}}+b_{2} \beta_{r r}^{-3}\left(1+\xi_{0}\right) e^{-\beta_{r r} \xi_{0}}+b_{3} \xi_{0}^{2} e^{-\beta_{r r} \xi_{0}}$,

where $b_{0}, b_{1}, b_{2}$, and $b_{3}$ are still unknown functions and

$$
\beta_{i j}^{2}=N_{i j}(a, \theta) h^{-3}(a, \theta) \text {. }
$$

The unknown functions can be related to the values of $w^{(0)}$ and $w^{(i)}$ and their derivatives at the outer and inner edges by using the boundary conditions.

Next, we note that $r=1$ corresponds to $\xi_{0}=0$ and $\xi_{i}$ $=(1-a) \epsilon^{-1}, \xi_{i} \rightarrow \infty$ as $\epsilon \rightarrow 0$. When $w=0$, Eqs. (13) and (33), yield

$$
w^{(m)}+c_{0}+\epsilon c_{4}=0 \text { at } r=1
$$

where

$$
c_{4}=c_{3}-\left(c_{1} / 2+\frac{5}{4} c_{2}\right) \lambda_{r r}^{-3} .
$$

When $\partial w / \partial r=0$, Eqs. (13) and (33), yield

$$
\epsilon \partial w^{(m)} / \partial r+\lambda_{r r}\left[c_{0}+\epsilon\left(c_{4}-c_{0} / 2\right)\right]=0 \text { at } r=1 .
$$

Eliminating the $c$ 's from Eqs. (35) and (36) gives

$$
w^{(m)}=\frac{\epsilon}{\lambda_{r r}(1-\epsilon / 2)} \frac{\partial w^{(m)}}{\partial r} \text { at } r=1 \text {. }
$$

The boundary conditions on $w$ at the inner edge can be transformed in a way similar to that used above for the outer edge. The result can be obtained from Eqs. (35) and (36) by simply replacing $\lambda_{0}$ with $\lambda_{i}$ and evaluating the result at $r=a$ instead of $r=1$.

Therefore, by either method, the problem of the transverse vibrations of highly prestressed circular or annular plates [Eqs. (2)-(5)] is reduced to the solution of an equivalent membrane problem consisting of the equation

$$
\mathcal{L}\left(w^{(m)}\right)=0,
$$

where the operator $\mathscr{L}$ is defined in Eq. (7), subject to one of the modified boundary conditions (27) in the case of the method of matched asymptotic expansions and (37) in the case of composite expansions. Thus, instead of solving a fourth-order differential equation subject to four boundary conditions, we reduced the problem to the solution of a second-order differential equation subject to two boundary conditions. The accuracy of our approach is demonstrated in the next section by comparing the present results with the exact solutions for a circular plate.

\section{COMPARISON OF EXACT AND PERTURBATION SOLUTIONS}

Reasonably accurate solutions to the transverse vibrations of moderately prestressed, clamped circular 
plates are available. ${ }^{1}$ However, solutions when such plates are highly prestressed are not directly available, but can be obtained by solving the relevant characteristic equations ${ }^{1}$ by the use of a Newton-Raphson technique. Such solutions will be treated as being the exact solutions for evaluating the accuracy of our new approach.

\section{A. Method of matched asymptotic expansions}

In the case of uniformly thick, uniformly prestressed plates, the operator of Eq. (25) becomes the Laplacian operator $\nabla^{2}$, and Eqs. (28) simplify to

$$
\begin{aligned}
& \nabla^{2} \phi_{0}+\lambda_{0} \phi_{0}=0, \\
& \nabla^{2} \phi_{1}+\lambda_{0} \phi_{1}=-\lambda_{1} \phi_{0} .
\end{aligned}
$$

These equations have to be solved subject to boundary conditions (27).

The solution of Eq. (39) subject to boundary condition (27a) can be rewritten as

$$
\phi_{0}=b J_{m}\left(\kappa_{m n} r\right) e^{(i m \theta)} \text {, }
$$

where $\kappa_{m n}$ is the root of

$$
J_{m}(\kappa)=0,
$$

and $b$ is chosen such that

$$
b^{2} \int_{0}^{1} r J_{m}^{2}\left(\kappa_{m n} r\right) d r=1 \text { or } b^{2}=2\left[J_{m}^{\prime}\left(\kappa_{m n}\right)\right]^{-2} .
$$

Since the homogeneous Eqs. (39) and (27a) have a nontrivial solution, the nonhomogeneous equations have a solution if, and only if, a solvability condition is satisfied which furnishes the value of $\lambda_{1}$. To determine this solvability condition, we express $\phi_{1}$ as

$$
\phi_{1}(r, \theta)=\sum_{s=-\infty}^{\infty} \psi_{s}(r) e^{(i s \theta)} .
$$

Substituting for $\phi_{1}$ and $\phi_{0}$ into Eqs. (40) and (27b), we have

$$
\begin{aligned}
& \sum_{s=-\infty}^{\infty}\left[\psi_{s}^{\prime \prime}+r^{-1} \psi_{s}^{\prime}+\left(\kappa_{m n}^{2}-s^{2} / r^{2}\right) \psi_{s}\right] e^{(i s \theta)} \\
& =-\lambda_{1} b J_{m}\left(\kappa_{m n} r\right) e^{(i m \theta)}, \\
& \sum_{s=-\infty}^{\infty} \psi_{s}(1) e^{(i s \theta)}=b \kappa_{m n} J_{m}^{\prime}\left(\kappa_{m n}\right) e^{(i m \theta)} .
\end{aligned}
$$

Multiplying Eqs. (44) and (45) by $\exp (-i m \theta)$ and integrating the results from $\theta=0$ to $\theta=2 \pi$, we obtain

$$
\begin{aligned}
& \psi_{m}^{\prime \prime}+r^{-1} \psi_{m}^{\prime}+\left(\kappa_{m n}^{2}-m^{2} r^{2}\right) \psi_{m}=-\lambda_{1} b J_{m}\left(\kappa_{m n} r\right), \\
& \psi_{m}(1)=b \kappa_{m n} J_{m}^{\prime}\left(\kappa_{m n}\right) .
\end{aligned}
$$

The problem of determining the solvability condition of Eqs. (41) and (27b) is thus reduced to that of determining the solvability condition of Eqs. (46) and (47). To accomplish this, we multiply Eq. (46) by $r J_{m}\left(\kappa_{m n} \gamma\right)$, integrate the result by parts from $r=0$ to $r=1$, use Eq. (47), and obtain

$$
\lambda_{1}=\kappa_{m n}^{2} b^{2}\left[J_{m}^{\prime}\left(\kappa_{m n}\right)\right]^{2}=2 \kappa_{m n}^{2} .
$$

Hence,

$$
\lambda=\kappa_{m n}^{2}(1+2 \epsilon)+\cdots,
$$

and

$$
\alpha \omega_{m n}=\lambda^{1 / 2}=\kappa_{m n}(1+2 \epsilon)^{1 / 2}+\cdots \approx \kappa_{m n}(1+\epsilon)+\cdots \cdot
$$

\section{B. Method of composite expansions}

In the case of uniformly thick, uniformly prestressed, solid circular plates, Eq. (38) becomes

$$
\nabla^{2} w^{(m)}-\alpha^{2} \partial^{2} w^{(m)} / \partial t^{2}=0 .
$$

A general solution to this equation which satisfies the requirement of finiteness of $w^{(m)}$ at $r=0$ and periodicity of $w^{(m)}$ in $\theta$ is

$$
w^{(m)}=A J_{m}(\omega \alpha r) e^{(i m \theta)},
$$

where $\omega$ is the frequency of transverse vibration and $m$ is the wave number in the circumferential direction. The characteristic equation for the frequency, $\omega$, in this case follows from the modified boundary conditions on $w_{m}$, namely Eq. (37). In the case of uniformly thick, uniformly prestressed plates, Eq. (37) simplifies to

$$
w^{(m)}=\frac{\epsilon}{\left(1-\frac{1}{2} \epsilon\right)} \frac{\partial w^{(m)}}{\partial r} \text { at } r=1 .
$$

The corresponding characteristic equation in the case of a clamped plate is

$$
J_{m}(\omega \alpha)=\left[\epsilon /\left(1-\frac{1}{2} \epsilon\right)\right] J_{m}^{\prime}(\omega \alpha),
$$

which for small values of $\epsilon$ can be simplified to

$$
J_{m} \omega \alpha\left(1-\frac{\epsilon}{1-\frac{1}{2} \epsilon}\right)=0 \text {. }
$$

The frequencies of transverse vibrations of clamped circular plates are then to the first-order of approximation, given simply by

$$
\alpha \omega_{m n}=\kappa_{m n}\left(1-\frac{1}{2} \epsilon\right)\left(1-\frac{3}{2} \epsilon\right)^{-1},
$$

where $\kappa_{m n}$ is the $n$th zero of the Bessel function of the first kind and $m$ th order.

Table I shows a comparison of approximate, fundamental frequencies as predicted by the use of Eqs. (48) and (52) for a clamped plate with corresponding exact

TABLE I. Fundamental frequencies of a clamped circular plate.

\begin{tabular}{lllll}
\hline \hline & \multicolumn{3}{c}{$\omega\left(a^{*}\right)^{2}\left(\rho^{*} h^{*} / D^{*}\right)^{1 / 2}, m=0, n=1$} \\
$\phi=\frac{N^{*}\left(a^{*}\right)^{2}}{14.68 D^{*}}$ & & Approximate & Approximate & Percent \\
\cline { 2 - 4 } & Exact & MCE & MMAE & error \\
& Ref. 1 & [Eq. (52)] & Eq. (48) & (MCE) \\
\hline 2.0 & 17.774 & 16.3862 & 15.241 & 7.81 \\
3.0 & 20.411 & 19.0662 & 18.206 & 6.59 \\
4.0 & 22.704 & 21.4181 & 20.693 & 5.66 \\
5.0 & 24.758 & 23.5183 & 27.882 & 5.01 \\
6.81 & 28.05 & 26.8775 & 26.344 & 4.18 \\
10.0 & 32.995 & 31.8817 & 31.45 & 3.37 \\
12.0 & 35.715 & 34.6294 & 34.239 & 3.04 \\
14.0 & 38.224 & 37.1614 & 36.802 & 2.78 \\
16.0 & 40.567 & 39.5216 & 39.187 & 2.58 \\
$\mathbf{2 7 . 2 4}$ & $\mathbf{5 1 . 5 8}$ & $\mathbf{5 0 . 6 9 6 3}$ & 50.44 & 1.90 \\
\hline \hline
\end{tabular}


values. ${ }^{1}$ It can be seen from this table that, for values of $\epsilon$ as high as approximately 0.15 , the error in the fundamental frequency parameter is only of the order of $8 \%$ in the case of a clamped circular plate. We note that if Eq. (52) is expanded for small $\Sigma$, and terms up to $O(\epsilon)$ are included, the result agrees exactly with Eq. (48b) obtained by the method of matched asymptotic expansions. However, as shown in Table I, Eq. (52) without expansion is more accurate than Eq. (48).

\section{CONCLUDING REMARKS}

A numerical-perturbation technique is presented for the determination of the linear transverse vibrations of highly prestressed, nonuniform plates. The method of composite expansions is used to reduce the problem to that of solving the linear transverse vibrations of an equivalent nonuniform membrane subject to modified boundary conditions. Since for highly prestressed plates, a state of membrane deformation exists everywhere in the plate except in thin layers next to the plate edges, a finite-element method using plate elements is not optimal for this problem, owing to the large number of elements needed to accurately determine the state of deformation next to the plate edges. On the other hand, the higher the prestressed load is, the more accurate the results of the methods of matched asymptotic and composite expansions. Thus, the more difficult it gets to calculate the response by using numerical techniques, the easier it becomes to calculate the response by using the present technique.

${ }^{1}$ A. W. Leissa, "Vibrations of Plates," NASA SP-160 (1969) (unpublished).

${ }^{2} \mathrm{O}$. C. Zienkiewicz, The Finite Element Method in Engineering Science (McGraw-Hill, New York, 1971).

${ }^{3}$ A. H. Nayfeh, Perturbation Methods (Interscience, New York, 1973).

${ }^{4}$ G. J. Efstathiades, J. Sound Vib. 16, (2) 231-253 (1971).

${ }^{5}$ W. E. Alzheimer and R. T. Davis, J. Eng. Mech. Div., Proc. ASCE 94, EM4, 905-917 (1968).

${ }^{6}$ W. B. Frazer, Int. J. Solids Struct. 11, 501-518 (1975). 